MARCH 2020

閍TEXAS

CO Dissociation on Model

$\mathrm{Co} / \mathrm{SiO}_{2}$ Catalysts - The Effect of Adsorbed Hydrogen

MICHAEL FLOTO

Mullins Lab 


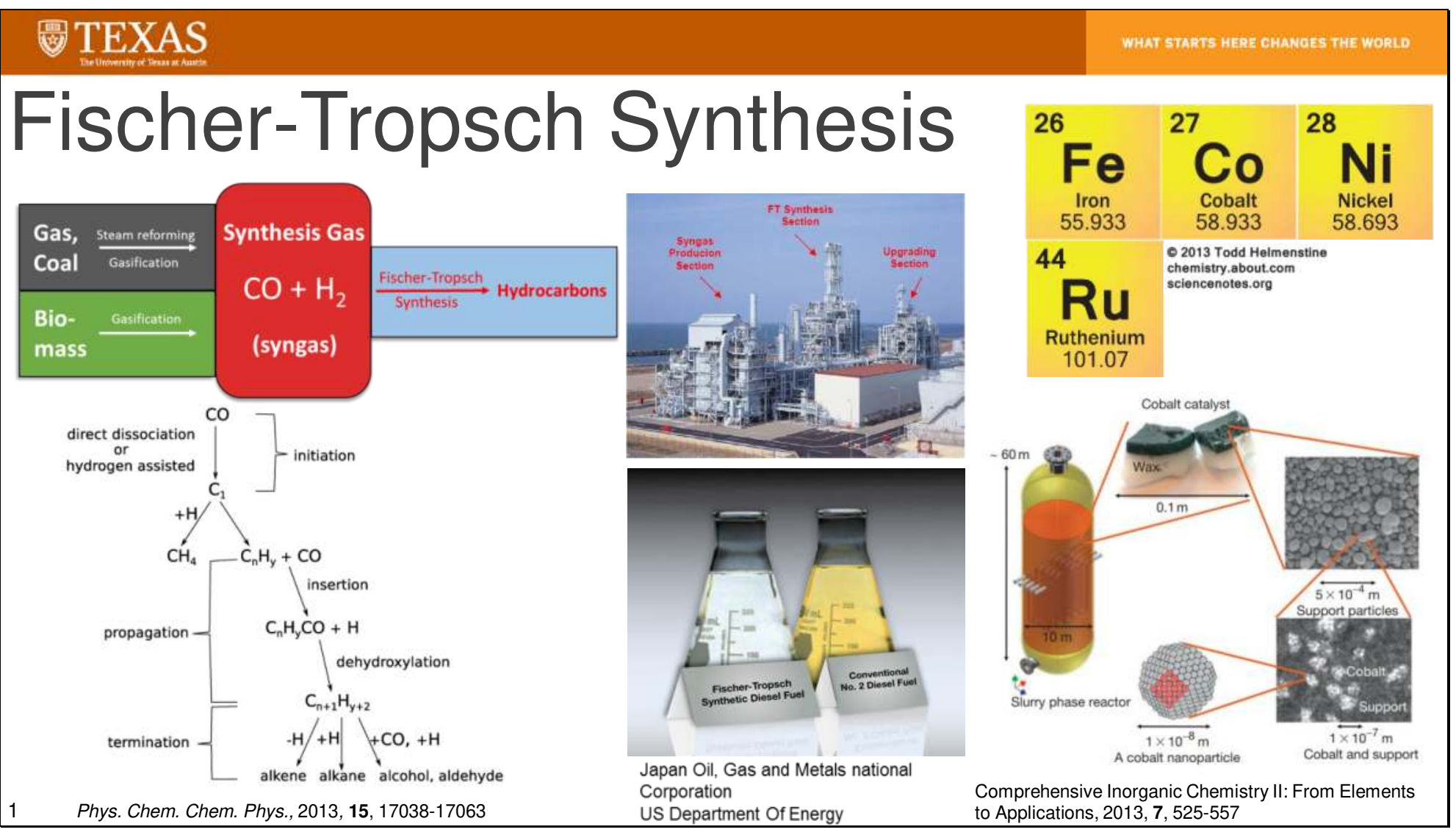

1. Fischer-Tropsch Synthesis (FTS) involves converting a mixture of carbon monoxide $(\mathrm{CO})$ and hydrogen $\left(\mathrm{H}_{2}\right)$ gas, known as synthesis gas, to a mixture of hydrocarbons to be used as chemical feedstock or fuels.

2. Implemented on an industrial scale, FTS offers a more sustainable source of energy relevant products, as the source of synthesis gas is often natural gas, coal, or biomass, allowing the mitigation of the need for petroleum based sources.

3. Transition metals such as iron, cobalt, nickel, and ruthenium, are active for FTS, with cobalt and iron most widely used in industry due to activity/selectivity and relative abundance.

4. This study will focus on cobalt catalysts for FTS, which mostly exist as nanoparticles supported on high surface area metal oxides, such as $\mathrm{SiO}_{2}$ (silica). 


\section{O: TEXAS}

WHAT STARTS HERE CHANOES THE WORLD

\section{Model studies vs Industrial catalysts}

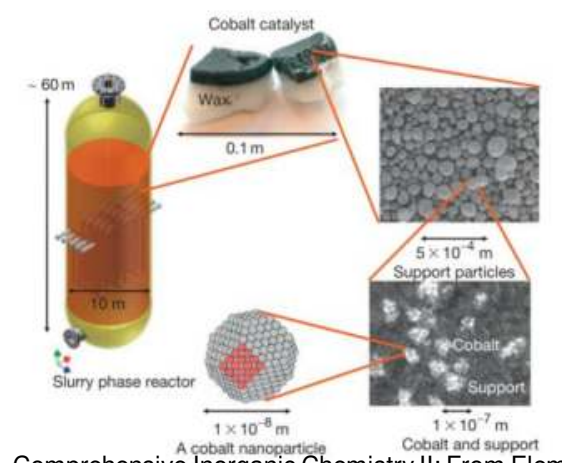

Comprehensive Inorganic Chemistry II: From Elements to Applications, 2013, 7, 525-557

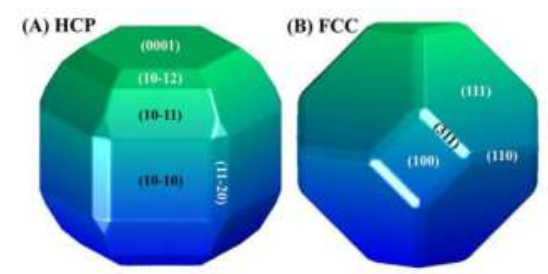

Figure 1. Equilibrium morphology of (A) HCP Co and (B) FCC Co based on the Wulff construction from DFT.

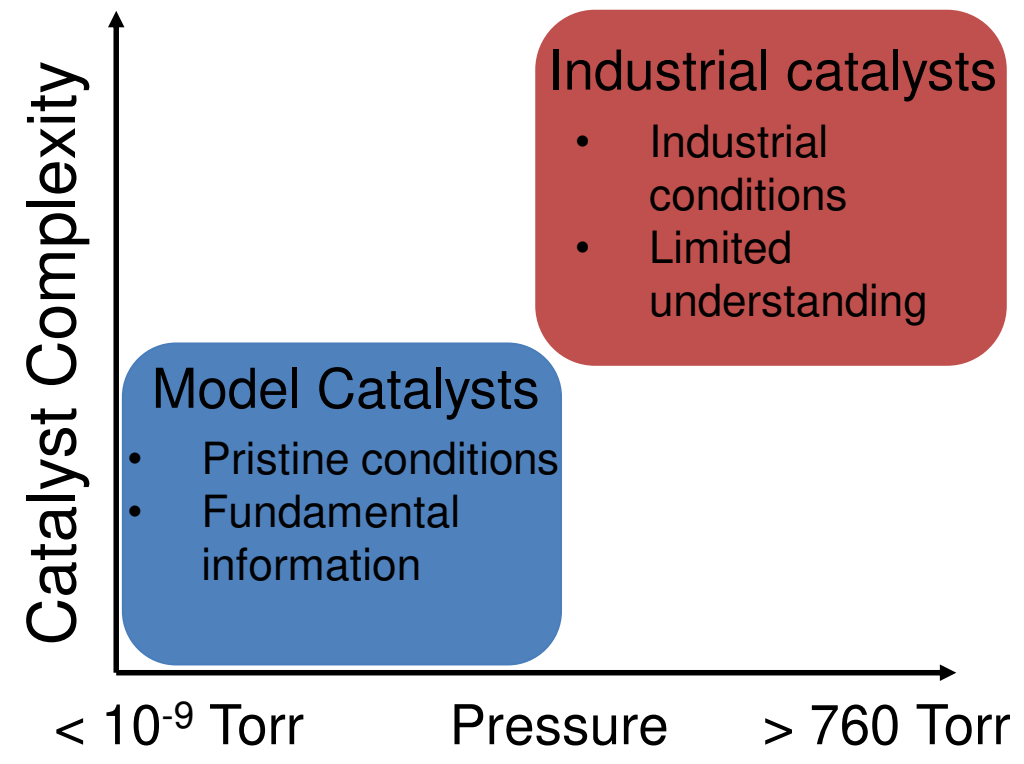

1. Model studies involve very powerful techniques that can reveal fundamental information about catalysis, but the impact of this information can suffer from a large pressure and materials gap to industrial catalysis.

2. Those gaps can be bridged by either conducting studies under higher pressure and/or on surfaces with increased complexity. 


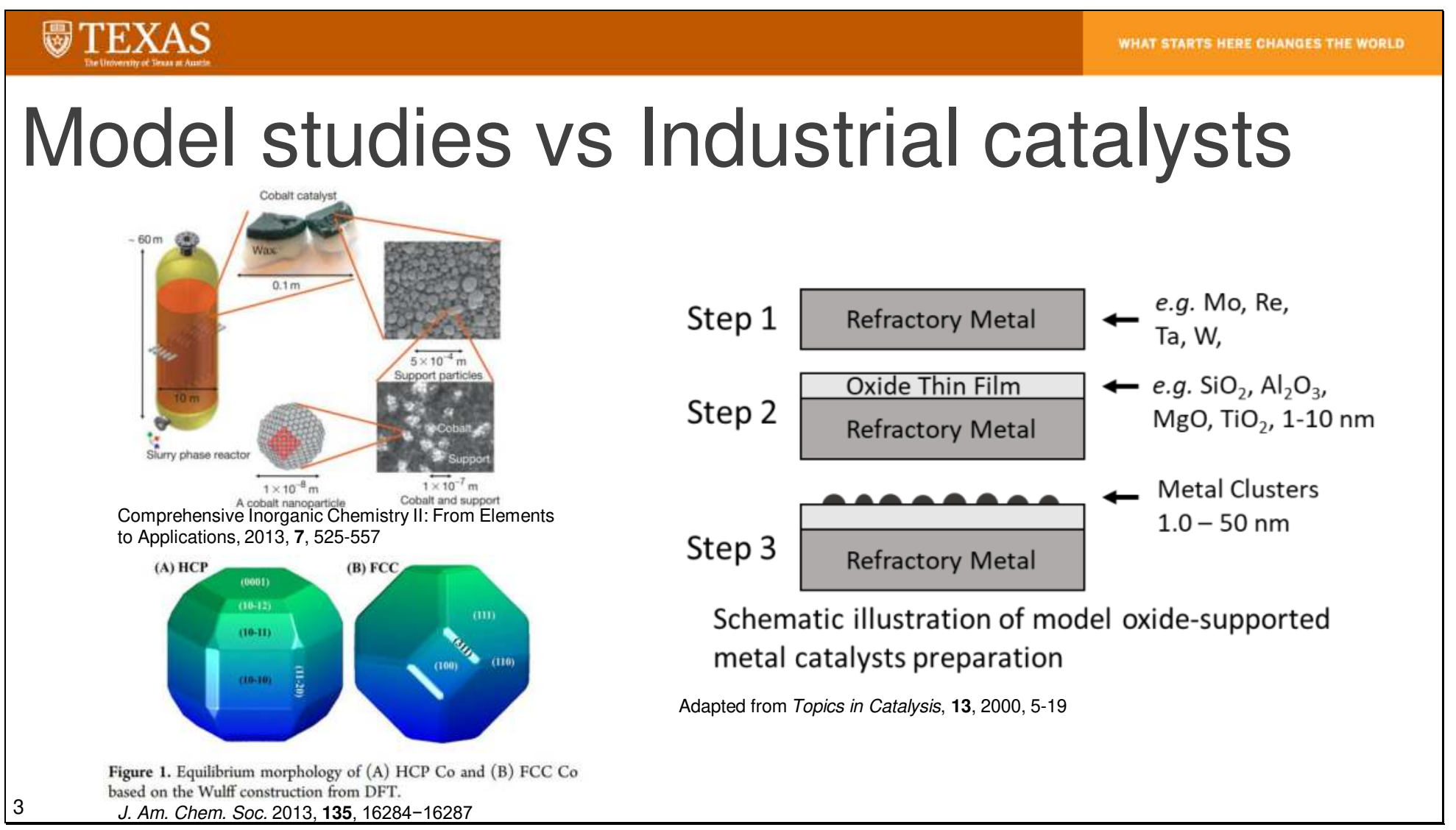

1. One method of increasing the complexity of a model surface that has been proven to be effective involves constructing a metal oxide film upon which metal clusters can be deposited. This technique usually involves physical vapor deposition (PVD) of silicon, aluminum, etc., onto a refractory metal support and then PVD of a transition metal onto the support. 


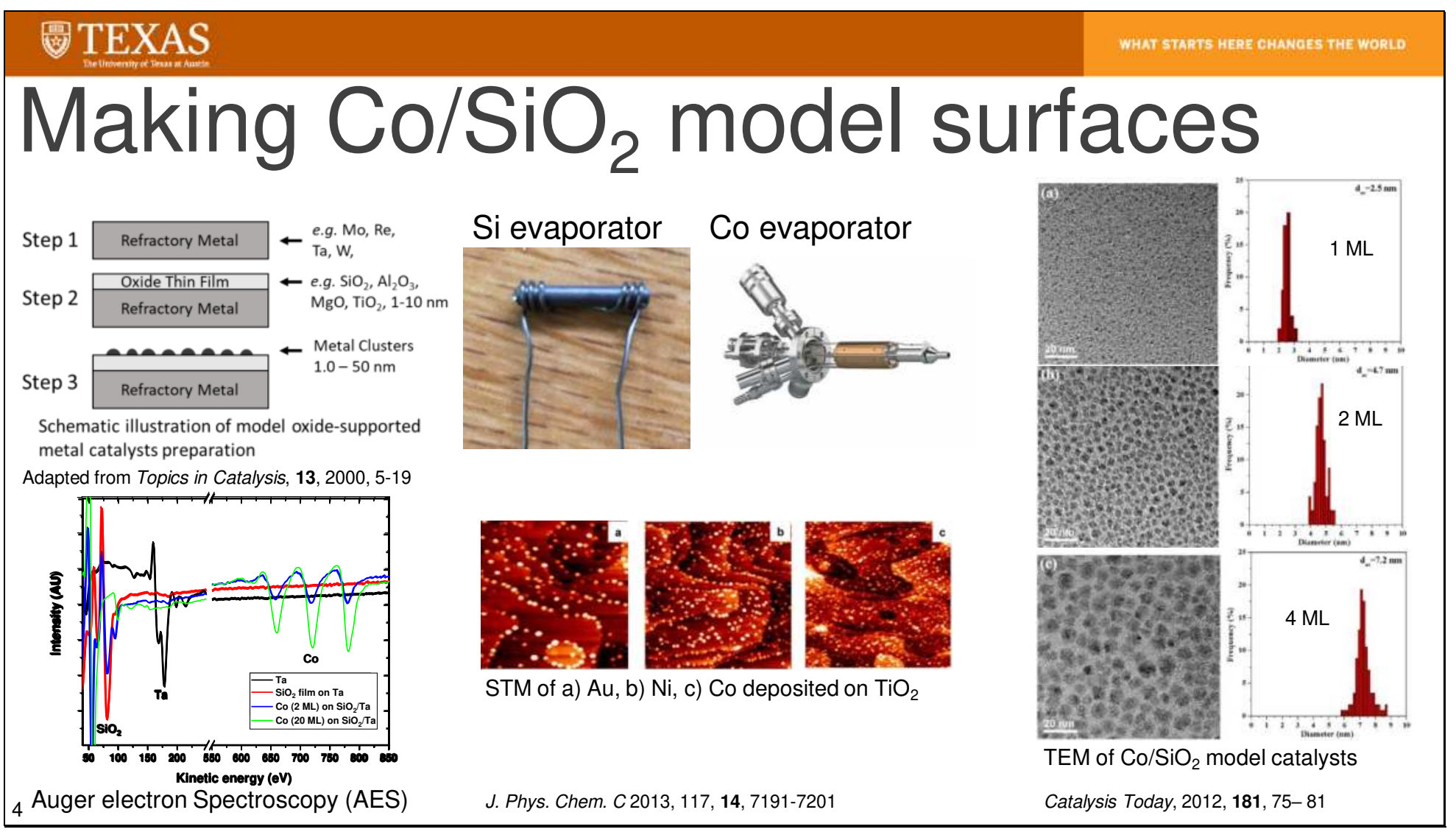

2. This technique has reliably shown to result in metal particles deposited onto a metal oxide support.

3. We replicated the PVD techniques used in work done by Goodman et al. studying FTS on model $\mathrm{Co} / \mathrm{SiO}_{2}$ surfaces.

4. We made Auger measurements that showed the deposition of cobalt on a silica film at room temperature combined with annealing resulted in particle formation of cobalt, in agreement with previous studies.

5. Transmission electron microscopy (TEM) measurements made by Goodman et al. showed deposition of 2 mono layers (ML) of cobalt combined with annealing resulted in cobalt particle size of about $5 \mathrm{~nm}$. 


\section{TEXAS

Temperature programmed desorption (TPD)

- $\mathrm{CO}$ on $\mathrm{Co} / \mathrm{SiO}_{2}$

- Expose Co film (20 ML) and Co nanoparticles (2 $\mathrm{ML}$ ) at room temperature to 1 Langmuir ( $\mathrm{L}$ ) of $\mathrm{CO}$ or $1 \mathrm{~L} \mathrm{H}_{2}+1 \mathrm{LCO}$, then heat to $900 \mathrm{~K}$

- Co film:

- Molecular CO desorption $375 \mathrm{~K}$, associative $\mathrm{CO}$ desorption $\sim 660 \mathrm{~K}$

- ${ }^{*} \mathrm{H}$ inhibits $\mathrm{CO}$ dissociation

- Co nanoparticles:

- Molecular CO desorption $375 \mathrm{~K}$, associative $\mathrm{CO}$ desorptions $\sim 800$

- ${ }^{*} \mathrm{H}$ enhances $\mathrm{CO}$ dissociation

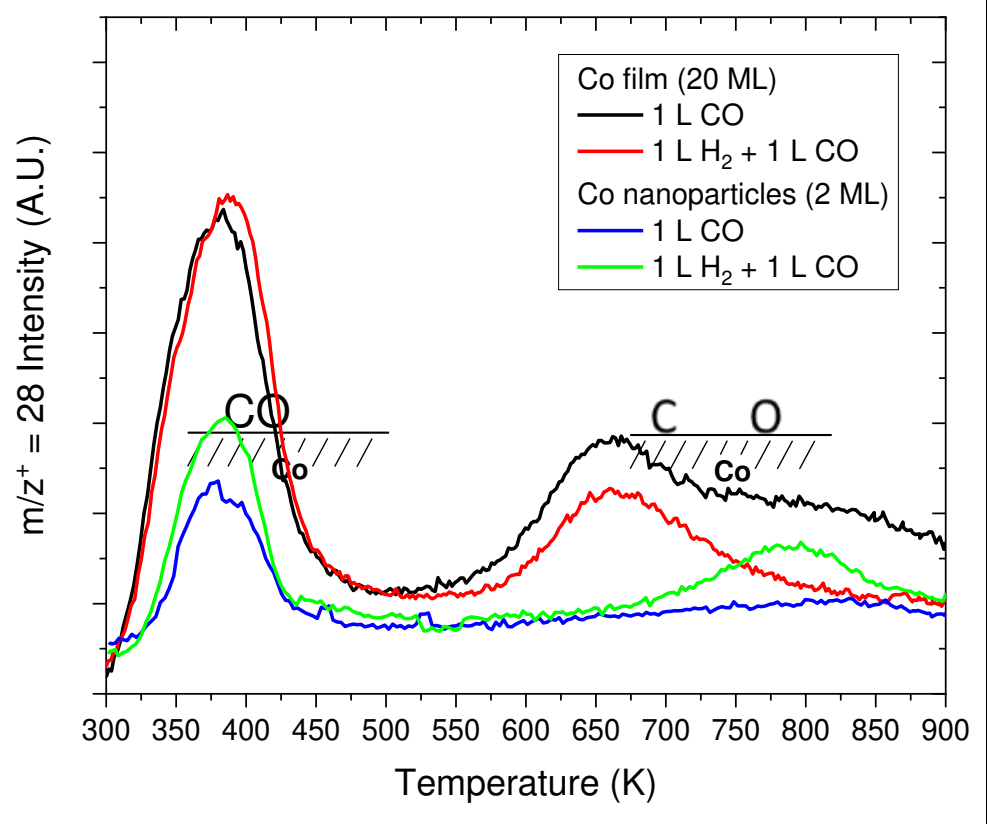

1. Temperature programmed desorption (TPD) experiments were conducted in order to study the crucial step of CO dissociation.

Note: $\mathrm{H}_{2}$ dissociates upon adsorption on cobalt at room temperature.

2. Adsorbed hydrogen $\left({ }^{*} \mathrm{H}\right)$ inhibits $\mathrm{CO}$ dissociation on the cobalt film, seen by a decrease of associative desorption. In contrast, ${ }^{*} \mathrm{H}$ enhances $\mathrm{CO}$ dissociation on cobalt nanoparticles.

3. The associative desorption feature for cobalt nanoparticles $(800 \mathrm{~K})$ is at a higher temperature than that of cobalt films $(660 \mathrm{~K})$. We argue this to be caused by the fact that cobalt nanoparticles are more readily oxidized than bulk cobalt. This suggests that $\mathrm{O}^{*}$ could be more strongly bound to the Co nanoparticles, causing associative desorption of $\mathrm{CO}$ to require more energy, compared with the cobalt films. 


\section{TEXAS

\section{FCC vs. HCP cobalt}

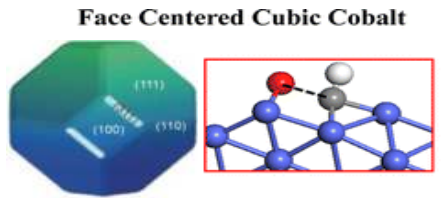

Hexagonal Close Packed Cobalt

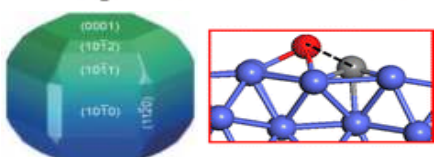

Activity
H-assisted Route

$$
\text { Low High } \quad \text { Direct Route }
$$

J. Am. Chem. Soc. 2013, 135, 16284-16287

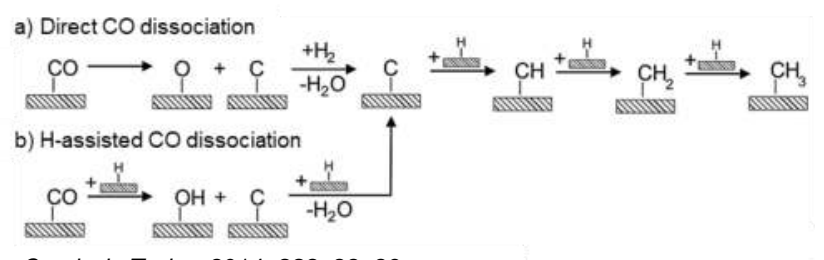

Catalysis Today, 2014, 228, 32-39

- Calculations have shown $\mathrm{CO}$ dissociation mechanism preference based on cobalt structure

- Cobalt particles $(<20 \mathrm{~nm})$ have shown to exist as mostly face-centerd-cubic (FCC) cobalt as apposed to hexagonal-closed-packed (HCP) cobalt

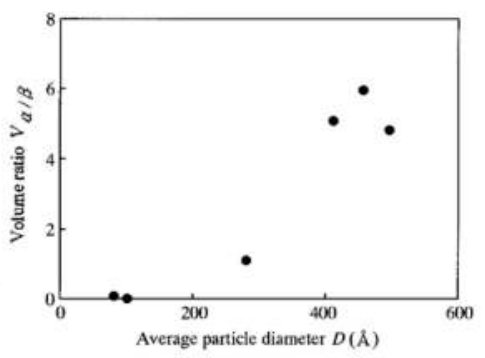

FIG. 1. Particle size dependence of volume ratio of hep $(\alpha-)$ to fec $\left(\beta\right.$-) phase $V_{\alpha / \beta}$ for Co fine particles. It is clearly noted that $\alpha$-phase becomes dominant with increase in the particle diameter D.

Phys. Rev. B., 1997, 56, 849-854

1. Based on calculations, $\mathrm{CO}$ dissociation has shown to be more facile through the hydrogen-assisted dissociation mechanism on FCC cobalt catalyst and more facile through the direct dissociation mechanism on HCP cobalt.

2. Under standard conditions, HCP is the thermodynamically favorable structure of bulk cobalt. At $700 \mathrm{~K}$, the FCC structure is more favorable and a phase shift occurs. Small cobalt particles $(<20 \mathrm{~nm})$ have reliably shown to exist in the form of FCC cobalt. This is thought to be due to the thermal energy involved in PVD and the strain effects caused by smaller particles. 


\section{(:) TEXAS}

FCC vs. HCP cobalt

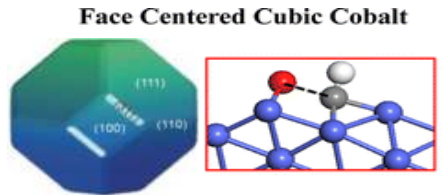

H-assisted Route
Hexagonal Close Packed Cobalt

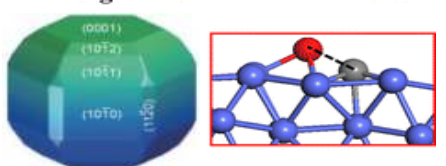

Activity

Direct Route

J. Am. Chem. Soc. 2013, 135, 16284-16287

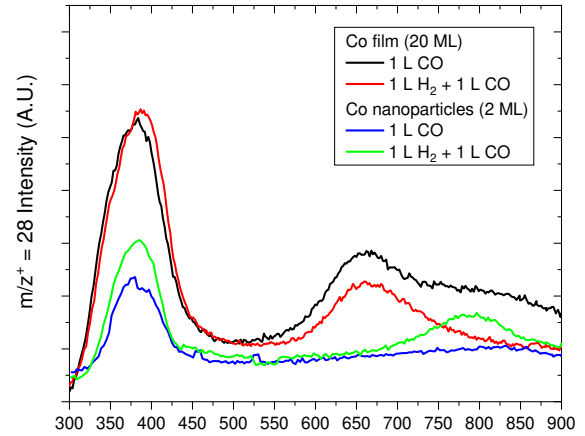

Temperature (K)

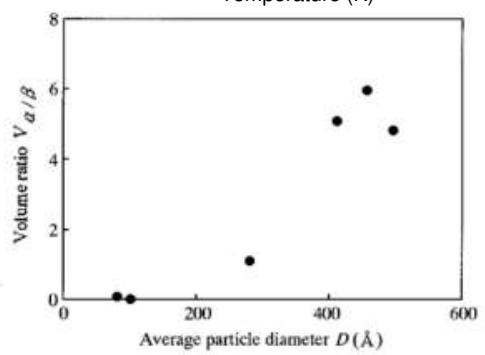

FIG. 1. Particle size dependence of volume ratio of hep ( $\alpha-)$ to fec ( $\beta$-) phase $V_{\alpha / \beta}$ for Co fine particles. It is clearly noted that $\alpha$-phase becomes dominant with increase in the particle diameter D. 


\section{Conclusions

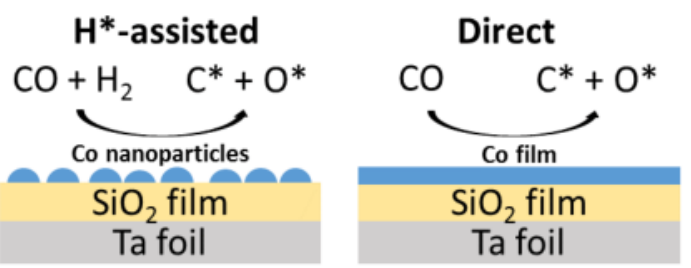

- Cobalt film (20 ML), ${ }^{*} \mathrm{H}$ inhibits $\mathrm{CO}$ dissociation, direct dissociation is preferred

- Cobalt nanoparticles (2 ML, annealed), *H enhances CO dissociation, hydrogen-assisted dissociation preferred

- Supported by DFT, as cobalt nanoparticles (<20 nm) are mostly FCC cobalt and cobalt films/bulk are mostly HCP cobalt

- Future: high pressure experiments (bridge the pressure gap) 


\section{TEXAS}

\section{Acknowledgements}

- Principal Investigator: Buddie Mullins

- Mullins Group

- Catalysis Subgroup: Sungmin Han, Ryan Ciufo, Ehren Eichler, Yongtak Kwon

- Funding: DOE, The Welch Foundation
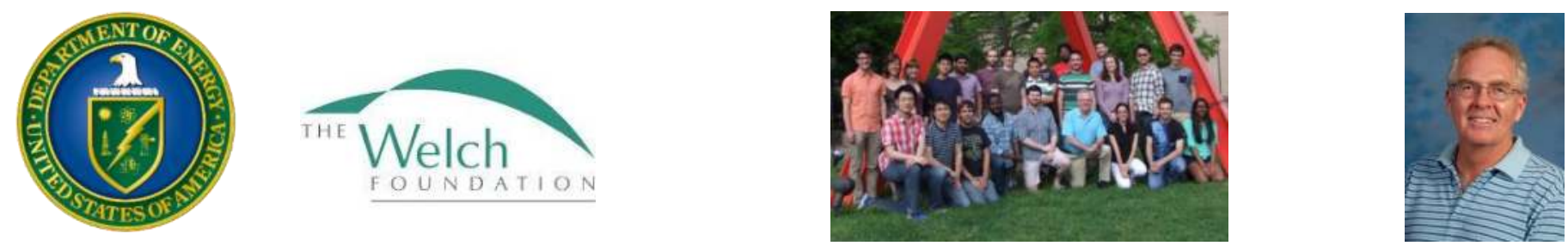